\title{
SATISFAÇÃO NO TRABALHO E CAPACIDADE PARA O TRABALHO ENTRE DOCENTES UNIVERSITÁRIOS
}

\author{
Elaine Cristina Marqueze* \\ Claudia Roberta de Castro Moreno"
}

\begin{abstract}
RESUMO. O objetivo principal deste estudo foi verificar a correlação entre satisfação no trabalho e capacidade para o trabalho de docentes universitários. Este é um estudo transversal de abordagem quantitativa. Dele fizeram parte 154 docentes, dos quais 50,6\% eram homens e 49,4\% eram mulheres e cuja idade média era de 39,25 anos. A coleta de dados incluiu três questionários: 1. Dados sociodemográficos e funcionais, 2. Escala de satisfação no trabalho; 3. Índice de capacidade para o Trabalho - ICT. A satisfação no trabalho e o ICT mostraram correlação estatisticamente significativa $(\mathrm{r}=0,23, \mathrm{p}<0,01)$. Conclui-se que o aumento da satisfação no trabalho pode melhorar a capacidade para o trabalho entre os docentes.

Palavras-chave: satisfação no trabalho, índice de capacidade para o trabalho, docente.
\end{abstract}

\section{JOB SATISFACTION AND WORK ABILITY AMONG COLLEGE EDUCATORS}

\begin{abstract}
The main aim of this study was to verify the correlation between job satisfaction and work ability among college educators. A quantitative approach was carried out in a cross-sectional design. The study included 154 educators, among them $50.6 \%$ were male and $40.4 \%$ female, with mean age of 39.25 years. Data collection included three questionnaires: 1. Socio-demographic and functional data; 2. Job satisfaction scale; 3. Work Ability Index -WAI. Job satisfaction and WAI showed a significant statistically correlation $(r=0.23, p<0.01)$. It can be conclude that the increase of job satisfaction can improve the educator's work ability.
\end{abstract}

Key words: Job satisfaction, work ability index, educator.

\section{SATISFACCIÓN EN EL TRABAJO Y CAPACIDAD PARA EL TRABAJO ENTRE DOCENTES UNIVERSITARIOS}

RESUMEN. El principal objetivo del presente estudio fue comprobar si hay correlación entre satisfacción en el trabajo y la capacidad para el trabajo de docentes universitarios. Se refiere a un estudio transversal de abordaje cuantitativo. Tomaron parte del estudio 154 docentes. La compilación de datos envolvió tres cuestionarios: 1. Datos socio-demográficos y funcionales, 2. Escala de satisfacción en el trabajo; 3. Índice de Capacidad para el Trabajo - ICT. La satisfacción en el trabajo y el ICT revelaron correlación estadística significante $(r=0,23, p<0,01)$. Se concluye que la satisfacción en el trabajo puede aumentar la capacidad para el trabajo entre los docentes.

Palabras-clave: Satisfacción en el trabajo; índice de capacidad para el trabajo; docente..

A satisfação no trabalho e a capacidade para o trabalho constituem fatores essenciais para os trabalhadores. Uma maior satisfação no trabalho, sem ansiedade e sem medo, faz com que o trabalhador encontre significado em sua atividade laboral e apresente atitudes positivas de enfrentamento à vida (Dejours, 1987; Seligmann-Silva, 1995).
Nos últimos 20 anos, pesquisadores de todo o mundo têm desenvolvido estudos com uma grande diversidade de categorias profissionais, os quais comprovam a participação do trabalho na geração de sofrimento psíquico (Gazzotti \& Codo, 2002). Nesse sentido, Oliveira (2002) afirma que a compreensão do trabalho envolve diretamente o estudo da atividade

* Doutoranda do Programa de Pós-Graduação da Faculdade de Saúde Pública da Universidade de São Paulo-USP.

\# Doutora em Saúde Pública. Professora do Departamento de Saúde Ambiental, Faculdade de Saúde Pública,Universidade de São Paulo-USP. 
cognitiva, pois esta revela os arranjos ergonômicos do sistema técnico-organizacional, bem como a carga de trabalho global.

Codo (1999) sugere que o trabalho tenha um papel na estruturação do indivíduo e na deflagração de prazer e sofrimento. Em sua opinião os problemas resultantes do sofrimento psíquico nas organizações são responsáveis por boa parte dos afastamentos do trabalho.

Nas universidades, têm-se encontrado, com raras exceções, processos dominantes de hierarquização e de burocratização (Wanderley, 1999), o que pode prejudicar o desenvolvimento das universidades, bem como a saúde dos docentes.

Gazzotti e Codo (2002) comentam que os docentes podem sofrer psicologicamente tanto por não darem atenção aos seus alunos como por dar-se atenção demasiada, o que por vezes é sentido como culpa. A exigência de concentração e o ritmo acelerado de trabalho também são aspectos psicológicos negativos freqüentemente citados por docentes (Paranhos, 2001).

Os diagnósticos mais freqüentes das licenças médicas são os de doenças musculoesqueléticas, distúrbios vocais e depressão (Esteve, 1999). Particularmente, no caso das mulheres, é observada maior frequiência de licenças médicas de diversas causas, com exceção das doenças digestivas e cardiovasculares, nas quais a maior prevalência é encontrada no grupo dos homens (Esteve, 1999). Além disso, as mulheres parecem ter maior comprometimento com sua saúde mental, dado que nem sempre utilizam vias compensatórias de recuperação da afetividade no trabalho (KhouryCarvalho, 1995).

Além dos problemas de saúde mental, outras queixas também foram referidas no estudo de Delcor et al. (2004), tais como as relacionadas à postura e ao uso intensivo da voz, mencionadas respectivamente por $50 \%$ e $45,7 \%$ dos docentes.

Esteve (1999) sugere que os principais fatores negativos e limitantes à prática docente referem-se à falta de recursos, problemas nas condições de trabalho, violência nas instituições e a acumulação de exigências sobre o professor. A insatisfação no trabalho e o desprestígio profissional estão entre os fatores que mais contribuem para que os professores deixem a docência (Lapo \& Bueno, 2003).

Não obstante, a produção científica que avalia as repercussões do trabalho docente na saúde física e psíquica destes profissionais ainda é incipiente, especialmente no Brasil, o que evidencia a necessidade de realizar mais estudos nessa área
(Esteve, 1999; Delcor et al., 2004; Reis, Carvalho, Araújo, Porto \& Silvany Neto, 2005). É nesse contexto que se insere o presente estudo, tendo como objetivo principal verificar a correlação entre satisfação no trabalho e capacidade para o trabalho de docentes universitários. Além disso, foram verificadas as relações entre capacidade para o trabalho e aspectos psicossociais da escala de satisfação no trabalho. Também foi objetivo do presente estudo investigar algumas relações entre capacidade para o trabalho e características socio-demográficas e funcionais, segundo área de atuação.

\section{METODOLOGIA}

\section{População}

Fizeram parte do presente estudo 154 docentes universitários. Somente foram incluídos na amostra os docentes que possuíam horas/aulas ministradas na época da coleta e tinham pelo menos um ano de trabalho docente na instituição. Foram excluídos os que estavam em licença (capacitação, doença, maternidade ou sem vencimentos) e os que possuíam apenas horas/aulas extraclasse.

Dos 154 participantes da pesquisa, $78(50,6 \%)$ eram do sexo masculino e $76(49,4 \%)$ do sexo feminino, com idade média de 39 anos e 3 meses $(\mathrm{DP}=8$ anos e 2 meses), variando entre 26 e 61 anos. O tempo médio de contratação na universidade era de 6 anos e 3 meses ( $D P=7$ anos e 4 meses).

A coleta de dados foi realizada após autorização do Comitê de Ética da Universidade do Extremo Sul Catarinense, de acordo com as normas da Resolução 196/96 do Conselho Nacional de Saúde.

\section{Instrumentos para coleta de dados}

$\mathrm{O}$ instrumento de coleta de dados foi composto de três questionários, conforme descrição abaixo.

Questionário 1 - Contém informações sobre as características sociodemográficas e funcionais dos docentes.

Questionário 2 - Escala de satisfação no trabalho do Occupational Stress Indicator - OSI. Este instrumento foi desenvolvido em 1988 por Robertson, Cooper e Willians (1990) e traduzido e validado para o português por Swan, Moraes e Cooper (1993). Essa escala utiliza vinte e dois diferentes aspectos psicossociais do trabalho por meio de escala de Likert de seis pontos, classificados em: (1) enorme insatisfação, (2) muita insatisfação, (3) alguma insatisfação, (4) alguma satisfação, (5) muita satisfação e (6) enorme satisfação. Esse instrumento é 
do tipo auto-aplicável, no qual o respondente autoavalia seus sentimentos em relação aos diferentes aspectos de trabalho, o que resulta em um escore total que varia de 22 a 132 pontos.

Pelo fato de a escala não possuir um ponto de corte definido nem divisão em níveis, determinou-se a seguinte categorização (Martinez, 2002):

1. insatisfação: enorme insatisfação e muita insatisfação;

2. satisfação intermediária: alguma insatisfação e alguma satisfação;

3. satisfação: muita satisfação e enorme satisfação.

No presente estudo, a escala de satisfação no trabalho mostrou correlação interna satisfatória, apresentando alfa de Cronbach de 0,93.

Questionário 3, Índice de Capacidade para o Trabalho - ICT, é um questionário do tipo autoaplicável, que avalia a capacidade física e mental para o trabalho (Tuomi, Ilmarinen, Jahkola, Katajarinne \& Tulkki, 1997a). Esse instrumento foi validado no Brasil em 1997 (Tuomi, Ilmarinen, Jahkola, Katajarinne \& Tulkki, 1997b).

O ICT é representado por um escore total que varia entre 7 e 49 pontos, categorizado em:

- de 7 a 27 pontos: capacidade para o trabalho ruim;

- de 28 a 36 pontos: capacidade para o trabalho moderada;

- de 37 a 43 pontos: capacidade para o trabalho boa;

- de 44 a 49 pontos: capacidade para o trabalho excelente.

Neste estudo o alfa de Cronbach do ICT no módulo completo foi de 0,54. No módulo do prognóstico da capacidade para o trabalho daqui a dois anos foi de 0,63 .

\section{Análise dos dados}

Os dados coletados foram tabulados e analisados por meio do programa EPI INFO6 do CDC - Centers for Disease Control \& Prevention de Atlanta (versão $6.04,2001$ ) e também do programa Statistica 6.0.

Para análise de correlação entre as variáveis quantitativas, foi realizado o teste de Spearman com nível de significância de $\mathrm{p}<0,05$; entre as variáveis nominais, utilizou-se o teste de associação do quiquadrado, também com nível de significância de $\mathrm{p}<0,05$.
Os docentes foram divididos em 3 áreas: saúde; outras áreas; saúde e outras áreas. Essa divisão foi realizada porque os docentes da área da saúde atuavam também em serviços de saúde, o que poderia influenciar sua capacidade para o trabalho. Para as análises de correlação e associação, segundo área de atuação, a categoria saúde e outras áreas foi excluída.

\section{RESULTADOS}

Grande parte $(46,1 \%)$ dos docentes entrevistados ministrava aula em mais de um curso de graduação na instituição pesquisada. A quantidade de horas de trabalho em sala de aula variava de 02 a 40 horas/aulas/semana, sendo que 64 docentes $(41,6 \%)$ lecionavam de 1 a 10 horas/aulas por semana, 70 docentes $(45,5 \%)$ de 11 a 20 horas/aulas por semana, 15 docentes $(9,7 \%)$, de 21 a 30 horas/aulas por semana e apenas 5 docentes $(3,3 \%)$ ministravam de 31 a 40 horas/aulas por semana.

Cento e vinte e sete docentes $(82,5 \%)$ possuíam horas/aulas extraclasse na instituição. A média de horas/aulas extraclasse era de 15,2 horas ( $\mathrm{DP}=12,2$ horas), sendo as mais frequientes as administrativas e as de orientação de trabalho de conclusão de curso (TCC) $-33,3 \%$ e $20,8 \%$ respectivamente.

Dos 154 docentes, $13(8,4 \%)$ ministravam aula na pós-graduação da própria instituição, de 15 a 64 horas/aulas por semestre.

A maioria dos docentes $(83,1 \%)$ trabalhava em mais de um turno na instituição, a qual funcionava em três turnos - manhã, tarde e noite. Dos 154 docentes, 116 possuíam outro emprego, sendo que 92 docentes $(79,3 \%)$ possuíam dois empregos, 22 (19\%) possuíam três, e 2 docentes $(1,7 \%)$ possuíam quatro empregos.

A variável satisfação no trabalho apresentou média de 99,2 pontos ( $\mathrm{DP}=13,1$ pontos), mediana de 101,5 pontos e moda de 107 pontos. Verificou-se que $55,1 \%$ dos docentes apresentam satisfação no trabalho, 41,5\% satisfação intermediária e 3,4\% insatisfação.

Os aspectos com os quais há maior proporção de docentes no nível de satisfação são: conteúdo do trabalho que realiza, relacionamento com outras pessoas na instituição e grau de motivação para o trabalho. Já os aspectos com maior proporção de docentes no nível de insatisfação foram: volume de trabalho que tem para desenvolver, grau de segurança (estabilidade) no emprego e grau em que a instituição absorve as potencialidades que julga ter (figura 1). 


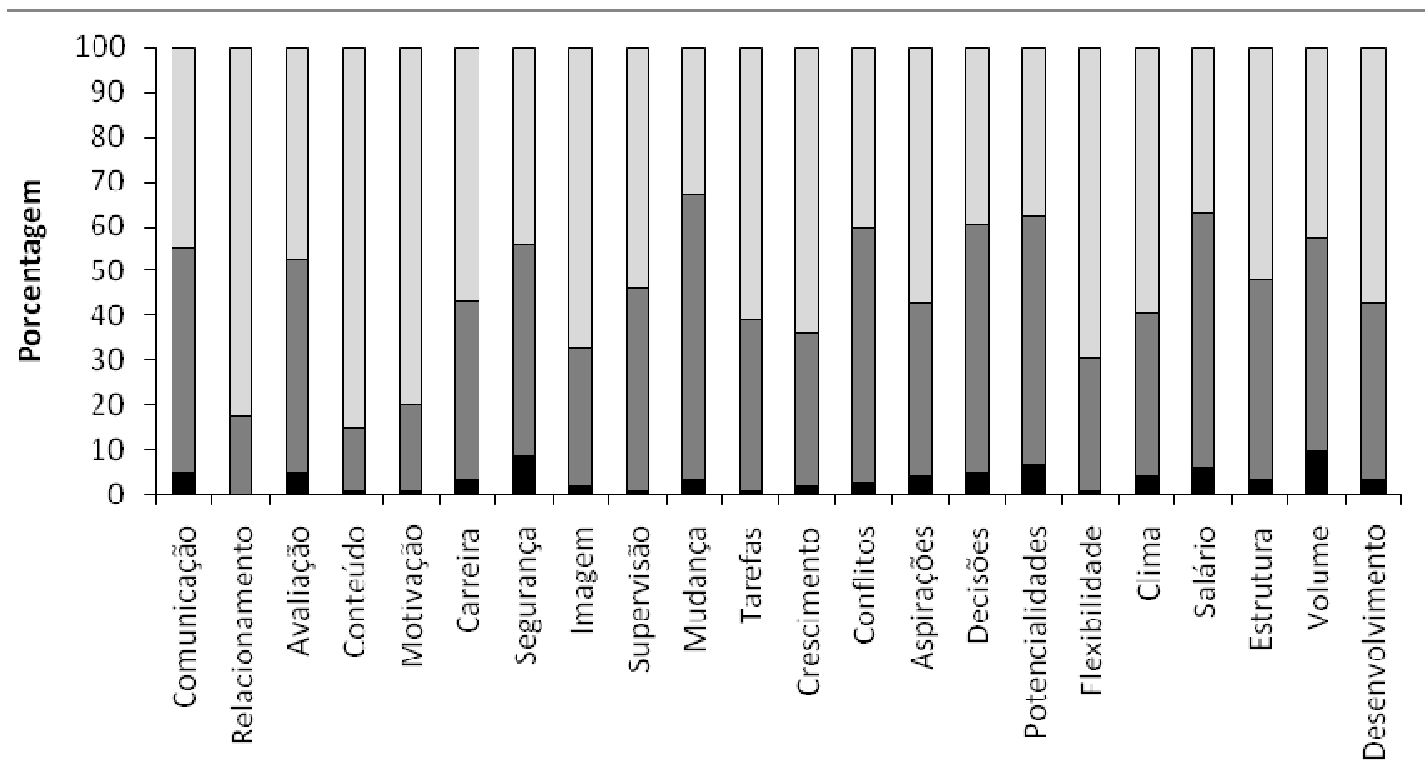

Aspectos psicossociais

- Insatisfação $\square$ Satisfaçãolntermediária $\square$ Satisfação

Figura 1. Porcentagem de Docentes, Segundo o Grau de Satisfação dos Aspectos Psicossociais do Trabalho

O escore médio do ICT dos docentes foi de 41,7 pontos ( $\mathrm{DP}=4,5$ pontos), o que representa boa capacidade para o trabalho. A maioria $(87 \%)$ dos docentes apresentava boa ou ótima capacidade para o trabalho (figura 2).

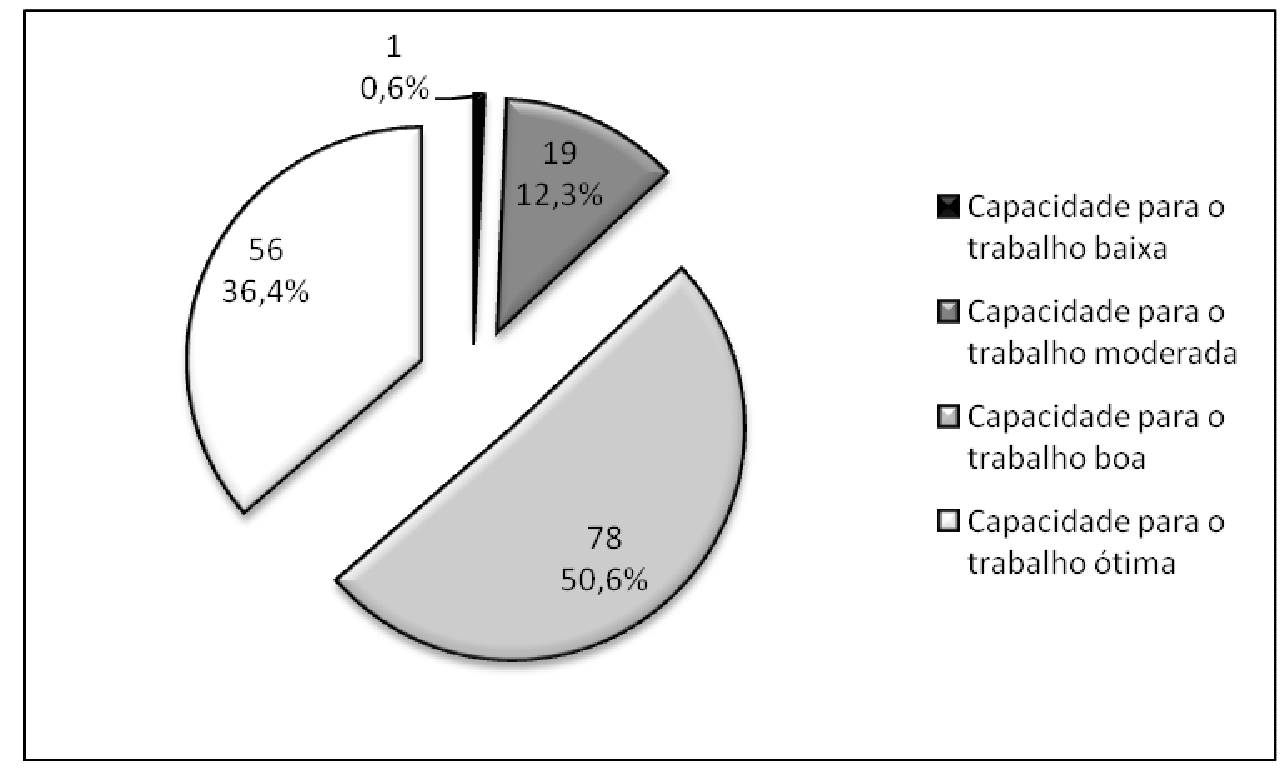

Figura 2.- Distribuição do Número e Porcentagem (\%) dos Docentes, Segundo o Índice de Capacidade para o Trabalho.

A maioria $(96,1 \%)$ dos docentes atribuiu nota superior a 7 pontos à capacidade atual para $o$ trabalho, quando comparada com a melhor que já teve em toda a sua vida (as notas vão de zero a 10 pontos).

Mais da metade dos docentes referiu pelo menos uma doença não diagnosticada $(64,3 \%)$. As doenças mais citadas foram as musculoesqueléticas, referidas por $69,5 \%$, desses docentes. Em seguida foram os distúrbios emocionais leves (mencionados por $27,3 \%$ dos docentes).

As doenças diagnosticadas pelo médico foram citadas por $61 \%$ dos docentes, sendo as musculoesqueléticas referidas por $53,9 \%$. As doenças 
respiratórias e as digestivas foram mencionadas por $26,0 \%$ e $20,8 \%$ dos docentes, respectivamente.

Menos de $20 \%$ dos docentes faltaram até 9 dias no ano anterior ao da pesquisa; no total o número de docentes que referiu faltas ao trabalho foi de 30 .

Em referência ao autoprognóstico sobre sua capacidade para o trabalho, a maioria $(86,4 \%)$ relatou que será capaz de continuar realizando seu trabalho nos próximos dois anos.

Não foi encontrada correlação entre horas/aulas ministradas e ICT entre todos os docentes estudados $(\mathrm{r}=-0,04, \mathrm{p}=0,54)$ e segundo área de atuação (saúde $\mathrm{r}=0,01, \mathrm{p}=0,88$; outras áreas $\mathrm{r}=-0,07, \mathrm{p}=0,55$ ).

Também não foi encontrada correlação entre horas/aulas extraclasse e ICT $(r=-0,07, p=0,32)$. Considerando-se a área de atuação, os dados também não mostraram correlação (saúde $\mathrm{r}=0,08, \mathrm{p}=0,51$; outras áreas $\mathrm{r}=0,17, \mathrm{p}=0,16$ ).

Realizando a soma das médias de horas/aulas ministradas com as de horas/aulas extraclasse, encontrou-se uma carga horária média de 29,1 horas $(\mathrm{DP}=12,5 \mathrm{~h})$. A carga horária total dos docentes, na instituição, também não apresentou correlação com o ICT ( $\mathrm{r}=-0,11, \mathrm{p}=0,17)$.

A variável idade também não apresentou correlação com o ICT $(\mathrm{r}=-0,03, \mathrm{p}=0,63)$, mesmo analisando-se os dados por área de atuação do docente (saúde $\mathrm{r}=-0,14, \mathrm{p}=0,25$ / outras áreas $\mathrm{r}=-0,08, \mathrm{p}=0,47$ ); ou seja, ter uma maior idade não implicou em ter um menor índice de capacidade para o trabalho.

Outra variável estudada foi o tempo de contratação dos docentes na referida instituição. Estar contratado há mais tempo pela instituição não está correlacionado a um menor ICT dos docentes ( $r=-$ $0,10, p=0,17)$, mesmo quando analisados segundo a área de atuação (saúde $\mathrm{r}=-0,24, \mathrm{p}=0,06$; outras áreas $\mathrm{r}=-0,17, \mathrm{p}=0,16)$.

Os resultados mostraram que não houve associação entre turno e índice de capacidade para o trabalho dos docentes $\left(\chi^{2}=0,58, p=0,44\right)$, ou seja, independentemente do turno de trabalho, o índice de capacidade para o trabalho não é modificado.

A variável sexo não estava associada ao ICT $\left(\chi^{2}=0,00, p=0,95\right)$, portanto, ser do sexo feminino ou do sexo masculino não modificou o índice de capacidade para o trabalho dos docentes, mesmo considerando-se a área de atuação (saúde $\chi^{2}=0,12$, $\mathrm{p}=0,72$; outras áreas $\chi^{2}=0,06, \mathrm{p}=0,81$ ).

Não foi encontrada associação entre ter outro emprego e o ICT, no entanto estava no limite de significância $\left(\chi^{2}=3,72, p=0,05\right)$. A associação desta variável com o ICT foi verificada somente entre os docentes da área da saúde $\left(\chi^{2}=4,91, \mathrm{p}=0,02\right)$, indicando que trabalhar em outros lugares pode vir a diminuir a capacidade para o trabalho destes docentes.

Encontrou-se correlação estatisticamente significativa entre satisfação no trabalho e índice de capacidade para o trabalho $(\mathrm{r}=0,23, \mathrm{p}=0,00)$. Portanto, quanto maior a satisfação em relação aos fatores psicossociais pesquisados, maior foi a capacidade em realizar o seu trabalho.

Ao se categorizar o grupo docente por sexo, foi encontrada correlação positiva entre satisfação no trabalho e ICT entre os docentes do sexo masculino $(r=0,30, p=0,00)$. Entre os docentes do sexo feminino não foi verificada correlação $(\mathrm{r}=0,15, \mathrm{p}=0,17)$.

No que se refere à correlação entre satisfação e ICT por área de atuação, os docentes da área da saúde apresentaram correlação positiva $(\mathrm{r}=0,46, \mathrm{p}=0,00)$, indicando que quanto mais satisfeitos estiverem, maior será o seu índice de capacidade para o trabalho. Entre os docentes de outras áreas não foi encontrada correlação $(r=0,08, p=0,49)$.

A análise dos aspectos psicossociais da escala de satisfação no trabalho mostrou que grande parte deles não está associada ao índice de capacidade para o trabalho (figura 3), quando cada um foi testado separadamente.

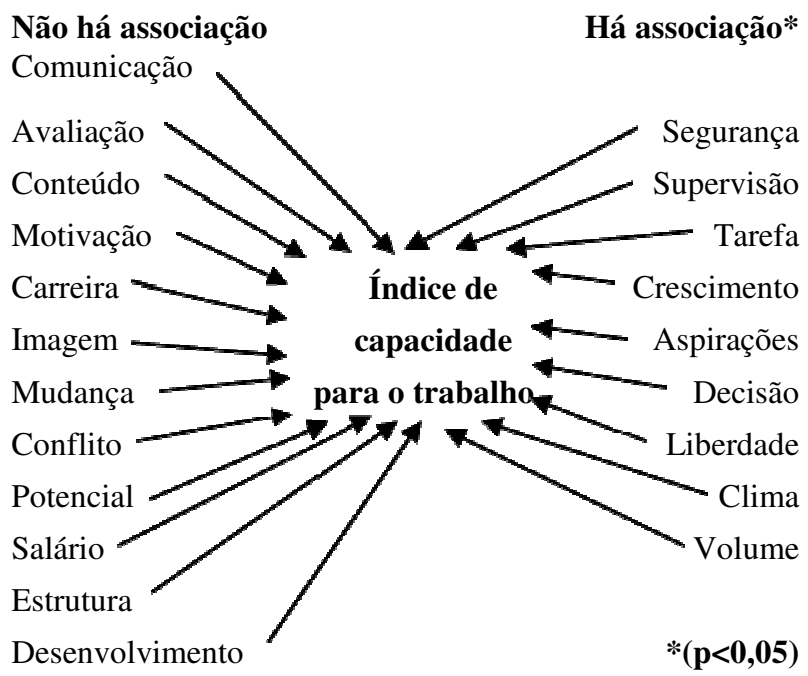

Figura 3. Aspectos Psicossociais da Escala de Satisfação no Trabalho Associados e Não Associados com o Índice de Capacidade para o Trabalho.

\section{DISCUSSÃO}

No presente estudo, a satisfação no trabalho e o índice de capacidade para o trabalho encontraram-se significativamente correlacionados, independentemente das variáveis sociodemográficas e 
funcionais, demonstrando a relevância dessas duas variáveis na saúde dos docentes. Tuomi et al. (1997a) e Bellusci e Fischer (1999) também encontraram esta correlação, sendo esta uma pré-condição para a saúde geral.

$\mathrm{Na}$ análise de cada um dos aspectos psicossociais foi encontrada associação entre os aspectos segurança, supervisão, tarefa, crescimento, aspirações, decisão, liberdade, clima e volume de trabalho.

No levantamento bibliográfico realizado, não foi encontrado nenhum estudo sobre a correlação destas duas variáveis em docentes; no entanto, em populações diferentes por exemplo, no estudo de Ramires, Graham, Richards, Cull e Gregory (1996) e de Martinez (2002) também foi encontrada associação significativa entre satisfação e capacidade para o trabalho, em que maiores níveis de satisfação estavam relacionados a uma melhor condição de saúde e à diminuição de queixas ou agravos à saúde.

Na variável sexo foi encontrada correlação entre satisfação no trabalho e o índice de capacidade para o trabalho somente entre os docentes do sexo masculino. Talvez o papel social da mulher na sociedade seja um fator determinante da satisfação ou não no trabalho; assim, por cumprir uma dupla jornada de trabalho (em casa e no trabalho), a capacidade do trabalho per se não constituiria um fator suficientemente importante para alterar a satisfação no trabalho, no caso das mulheres. Além disso, não foi observada associação entre sexo e ICT, apesar de Jacques e Codo (2002) afirmarem a necessidade da diferenciação na identidade psicológica do sexo, devido à identificação associada aos papéis femininos e à imagem de corpotrabalho. Tanto Bellusci e Fischer (1999) como Monteiro (1999) encontraram associação significativa entre sexo e ICT. No caso da atividade docente, o sexo, provavelmente, não influi no ICT, uma vez que as exigências laborais não diferem em relação a este fator.

A idade dos docentes não está correlacionada ao ICT, indo ao encontro do que afirmam Ilmarinen et al. (1991) e Shephard (2003), ou seja, que o envelhecimento funcional não está necessariamente relacionado ao envelhecimento cronológico, o qual depende das condições de vida e do trabalho, além das características individuais e do estilo de vida adotado. No caso, isto indica que os docentes têm boas condições de vida e de trabalho.

Segundo a área de atuação, foi observada correlação entre satisfação e índice de capacidade para o trabalho somente entre os docentes da área da saúde, evidenciando que os aspectos da saúde mental foram mais relevantes entre esses docentes. Jacques e Codo
(2002) observam que o campo da saúde é considerado de risco para problemas mentais no trabalho. Sendo assim, medidas preventivas em relação à satisfação no trabalho devem ser adotadas, prioritariamente entre os docentes da área da saúde. Uma hipótese que pode ser levantada a partir desta constatação é que os docentes da área da saúde, por trabalharem na assistência às pessoas, valorizam mais as questões relacionadas aos aspectos psicossociais.

Poucos docentes trabalhavam em apenas um turno na instituição, apesar de a maioria possuir outro(s) emprego(s), despendendo um tempo expressivo nele(s). Delcor et al. (2004) encontraram, entre os docentes pesquisados, $78,4 \%$ que trabalhavam em outra atividade remunerada, mostrando que entre os docentes não é raro encontrar trabalhadores que necessitam realizar outra atividade a fim de complementar a renda familiar. Vale ressalvar também que os docentes da área da saúde possuíam uma carga horária de trabalho maior em outros empregos, quando comparados aos docentes de outras áreas. Para muitos, a jornada de trabalho é longa, e isto, para Borges e Argolo (2002), pode refletir-se em sofrimento psíquico, por causa do elevado desgaste e da fadiga.

A maior parte dos docentes encontrava-se no nível de satisfação plena ou no nível de satisfação intermediária. Martinez (2002), em sua pesquisa, apresentou alguns estudos sobre satisfação no trabalho, ressaltando que não há um consenso na literatura sobre um valor de referência desta variável.

Entre os aspectos psicossociais referidos como os que mais causam insatisfação, destacou-se o volume de trabalho, comumente citado em outros pesquisadores, como Korunka e Vitouch (1999), os quais, em pesquisa realizada com professores, constataram que dentre as maiores fontes de insatisfação no trabalho está o excesso de trabalho a ser realizado em pouco tempo. É importante ressaltar que a atividade docente não se encerra na sala de aula, e o docente muitas vezes utiliza horas de lazer para realizar atividades de trabalho.

O grau de segurança (estabilidade) no emprego é outro aspecto que parece influenciar sobremaneira a satisfação no trabalho. Tanto Korunka e Vitouch (1999), Elovainio, Kivimãki, Steen e KalliomãkiLevanto (2000) e O’Driscoll e Beehr (2000) são unânimes em afirmar que a estabilidade no emprego contribui favoravelmente para a satisfação no trabalho.

Um aspecto que merece ser mais bem investigado é o grau em que a instituição absorve as potencialidades que o trabalhador julga ter. Este aspecto talvez gere insatisfação nos docentes, pela percepção de que poucas oportunidades lhes são 
ofertadas. O salário, ao contrário, é um aspecto já bem estudado. Para Locke (1976), além do valor real do dinheiro, este representa simbolicamente uma medida de competência no mercado de trabalho, em que mesmo quando justo, não é motivo de satisfação para as pessoas, a menos que seja suficiente para suprir suas despesas. Os estudos de Cavanagh (1992), Elovainio et al. (2000) e O'Driscoll e Beehr (2000), apesar de terem sido realizados em populações diferentes da estudada na presente pesquisa, verificaram associação estatisticamente significativa entre satisfação no trabalho e obtenção de bons salários e benefícios.

Parece não haver dúvidas quanto à relação entre relacionamento e satisfação no trabalho, também já observada em diversos outros estudos (Ramires et al., 1996; Korunka \& Vitouch, 1999; Elovainio et al., 2000; O'Driscoll \& Beehr, 2000). Já Cavanagh (1992) encontrou relação negativa entre a integração no trabalho e a satisfação no trabalho. No estudo de Ramires et al. (1996), realizado com médicos, foi identificado que a insatisfação no aspecto relacionamento estava associada ao esgotamento profissional, representando um fator importante para a saúde. Borges e Argolo (2002) constataram que fatores de prazer estão associados à satisfação no trabalho, o que também foi encontrado entre os docentes desta pesquisa.

A maioria dos docentes apresentou boa ou ótima capacidade para o trabalho, sendo que os homens apresentaram um ICT melhor que o das mulheres, o mesmo se verificando entre os docentes da área da saúde em relação aos de outra área. Um número expressivo de docentes não referenciou nenhuma lesão/doença diagnosticada pelo médico. Neste caso, o percentual foi maior do que o encontrado no estudo de Delcor et al. (2004) com professores da rede particular de ensino fundamental e médio (26,6\%). O'Driscoll e Beehr (2000) afirmam que uma maior satisfação remete a um menor número de queixas em relação à saúde, situação esta encontrada no presente estudo.

As lesões/doenças apontadas como as mais diagnosticadas foram as doenças musculoesqueléticas. Esteve (1999) e Delcor et al. (2004) afirmam que estas doenças são as mais freqüentes entre os docentes. Tuomi et al. (1997a) verificaram relação positiva entre as doenças musculoesqueléticas com a baixa capacidade para o trabalho.

Em seguida, estão as doenças respiratórias e as digestivas, que, segundo Delcor et al. (2004), também estão entre as mais freqüentes nessa classe ocupacional, sendo as digestivas mais freqüentes entre os homens.
Um número expressivo de referências bibliográficas (Khoury-Carvalho, 1995; Esteve, 1999; Gazzotti \& Codo, 2002; Delcor et al., 2004) sobre doenças em docentes refere-se a problemas de saúde mental. De maneira geral, os estudos dos autores supracitados referiram relação positiva entre diminuição da satisfação no trabalho e estresse dos docentes. Henne e Locke (1985) e Ramires et al. (1996) também apontam relação positiva entre essas duas variáveis: satisfação no trabalho e estresse dos docentes. A preocupação com a saúde mental torna-se essencial na área da saúde do trabalhador, visto que os prejuízos decorrentes dessa doença são altíssimos. Além deste fator, as ausências e afastamentos no trabalho, em grande proporção, são decorrentes de sofrimento psíquico (Jacques \& Codo, 2002). No caso dos docentes pesquisados, eles não associaram os aspectos citados como influenciadores negativos de sua capacidade para o trabalho, pois a maioria referiu um prognóstico positivo da sua capacidade de trabalho para daqui a dois anos.

Vale assinalar que este estudo apresentou como limitação o fato de a amostra ter sido constituída por $37,94 \%$ do total de 406 docentes da instituição, pois somente esse percentual concordou em participar da pesquisa.

\section{CONCLUSÃO}

A satisfação no exercício do trabalho docente pode aumentar a capacidade para o trabalho do profissional dessa área. Quanto aos aspectos psicossociais com maior proporção de docentes no nível satisfação, conclui-se que os docentes estão satisfeitos com a atividade realizada, assim como com o ambiente em que estão inseridos.

Quanto aos aspectos com maior proporção de docentes no nível insatisfação encontrados nesta pesquisa (volume de trabalho, grau de estabilidade no emprego e grau que a instituição absorve as potencialidades), conclui-se que eles são passíveis de reversão, pois referem-se a questões inerentes à condução do trabalho, que depende essencialmente dos gestores da universidade.

Especificamente para os docentes da área da saúde, a diminuição da capacidade para o trabalho poderia ser explicada pela atuação paralela dos docentes em serviços de saúde, que exige grande cabedal cognitivo. Neste contexto, salienta-se a importância da realização de estudos com docentes da área da saúde que investiguem a relação entre o exercício da docência e a prática de atividades em serviços de saúde. 


\section{REFERÊNCIAS}

Bellusci, S. M., \& Fischer, F. M. (1999). Envelhecimento funcional e condições de trabalho em servidores forenses. Revista de Saúde Pública, 33(6), 602-609.

Borges, L. O., \& Argolo, J. C. T. (2002). Estratégias organizacionais na promoção da saúde mental do indivíduo podem ser eficazes? Em M. G. Jacques \& W. Codo (Orgs.), Saúde mental \& trabalho: leituras (pp. 271-295). Petrópolis: Vozes.

Cavanagh, S. J. (1992). Job satisfaction of nursing staf working in hospitals. Journal of Advanced Nursing, 17(6), 704-711.

Codo, W. (1999). Educação: carinho e trabalho. Petrópolis: Vozes.

Dejours, C. (1987). A loucura do trabalho: estudo de psicopatologia do trabalho. São Paulo: Cortez/Oboré.

Delcor, N. S., Araújo, T. M., Reis, E. J. F. B., Porto, L. A., Carvalho, F. M., Oliveira \& Silva, M., Barbalho, L., \& Andrade, J. M. (2004). Condições de trabalho e saúde dos professores da rede particular de ensino de Vitória da Conquista, Bahia, Brasil. Cadernos de Saúde Pública, 20(1), 187-196.

Elovainio, M., Kivimãki, M., Steen, N., \& Kalliomãki-Levanto, T. (2000). Organizational and individual factors affecting mental health and job satisfaction: A multilevel analysis of job control and personality. Journal of occupational health psychology, 5(2), 269-277.

Esteve, J. M. (1999). O mal-estar docente: a sala de aula e a saúde dos professores (D. C. Cavicchia, Trad.). Bauru: EDUSC. (Trabalho original publicado em 1997)

Gazzotti, A., \& Codo, W. (2002). Histeria: doença profissional. Em M. G. Jacques \& W. Codo (Orgs.), Saúde mental \& trabalho: leituras (pp. 342-400). Petrópolis: Vozes.

Henne, D., \& Locke, E. (1985). Job Dissatisfaction: What are the consequences? International Journal of Psychology, 20, 221-240.

Ilmarinen, J., Tuomi, K., Eskelinen, L., Nygard, C. H., Huuhtanen, P., \& Klockars, M. (1991). Background and objectives of the finish research project on aging workers in municipal occupations. Scandinavian journal of work, environment \& health, 17(suppl. 1).

Jacques, M. G., \& Codo, W. (2002). Prefácio: uma urgência, uma busca, uma ética. Em M. G. Jacques \& W. Codo (Orgs.), Saúde mental \& Trabalho: leituras (pp. 7-16). Petrópolis, RJ: Vozes.

Korunka, C., \& Vitouch, O. (1999). Effects the implementation of information technology on employees strain and job satisfation: A context-dependent approach. Work and Stress, 34(4), 341-363.

Khoury-Carvalho, H. T. T. (1995). Professora primária: amor e dor. Em W. Codo \& J. J. C. Sampaio (Orgs.), Sofrimento psíquico nas organizações - Saúde mental e trabalho (pp. 127-138). Petrópolis: Vozes.

Lapo, F. R., \& Bueno, B. O. (2003). Professores, desencanto com a profissão e abandono do magistério. Cadernos de Pesquisa, 118, 65-88.

Locke, E.A. (1976). The nature and causes of job satisfaction. Em M. D. Dunnette (Ed.), Handbook of industrial and organizational psychology (pp. 1297-1349). Chicago: Rand McNally.
Martinez, M. C. (2002). As relações entre a satisfação com aspectos psicossociais no trabalho e a saúde do trabalhador. Dissertação de Mestrado não publicada, Programa de Pós-Graduação em Saúde Pública, Departamento de Saúde Ambiental, Faculdade de Saúde Pública, Universidade de São Paulo.

Monteiro, M. S. (1999). Envelhecimento e capacidade para o trabalho entre trabalhadores brasileiros. Tese de Doutorado não publicada, Programa de Pós-Graduação em Saúde Pública, Departamento de Saúde Ambiental, Faculdade de Saúde Pública, Universidade de São Paulo.

O'Driscoll, M. P., \& Beehr, T. A. (2000). Moderating effects of perceived control and need for clarity on the relationship between role stressors and employee affective reactions. The Journal of Social Psychology, 140(2), 151-159.

Oliveira, P. A. B. (2002). Trabalho coletivo: a construção de espaços de cooperação e de trocas cognitivas entre os trabalhadores. Em M. G. Jacques \& W. Codo (Orgs.), Saúde mental \& Trabalho: leituras (pp. 82-97). Petrópolis: Vozes.

Paranhos, I. (2001). Interface entre trabalho docente e saúde dos professores da Universidade Estadual de Feira de Santana. Dissertação de Mestrado não publicada, Programa de PósGraduação em Saúde Coletiva, Departamento de Saúde, Universidade Estadual de Feira de Santana.

Ramires, A. J., Graham, J., Richards, M. A., Cull, A., \& Gregory, W. M. (1996). Mental health of hospital consultants: The effects of stress and satisfaction at work. The Lancet, 347(9003), 724-728.

Reis, E. J. F. B., Carvalho, F. M., Araújo, T. M., Porto, L. A., \& Silvany Neto, A. M. (2005). Trabalho e distúrbios psíquicos em professores da rede municipal de Vitória da Conquista, Bahia, Brasil. Cadernos de Saúde Pública, 21(5), 1480-1490.

Robertson, I. T., Cooper, C. L., \& Willians, J. (1990). The validity of the occupational stress indicator. Work and Stress, 4(1), 29-39.

Seligmann-Silva, E. (1995). Psicopatologia e psicodinâmica no trabalho. Em R. Mendes (Org.), Patologia do trabalho (pp. 278310). Belo Horizonte: Atheneu.

Shephard, R.J. (2003). Envelhecimento, atividade física e saúde. São Paulo: Phorte.

Swan, J. A., Moraes, L. F. R., \& Cooper, C. L. (1993). Developing the occupational stress indicator (OSI) for use in Brazil: A report on the reliability and validity of the translated OSI. Stress Medicine, 9, 247-253.

Tuomi, K., Ilmarinen, J., Jahkola, A., Katajarinne, L., \& Tulkki, A. (1997a). Work ability index. Finnish: Institute of occupational health Helsinki.

Tuomi, K., Ilmarinen, J., Jahkola, A., Katajarinne, L., \& Tulkki, A. (1997b). Índice de Capacidade para o trabalho (F. M. Fischer, Trad.). Finlândia: Instituto de saúde ocupacional de Helsinki.

Wanderley, L. E. W. (1999). O que é universidade. São Paulo: Brasiliense.

Recebido em 12/07/2007 Aceito em 25/04/2008

Endereço para correspondência : Claudia Roberta de Castro Moreno, Departamento de Saúde Ambiental, Faculdade de Saúde Pública, USP, Av. Dr. Arnaldo, 715, CEP 01246-904, São Paulo-SP, Brasil. E-mail: crmoreno@usp.br 